Research Paper

\title{
A Novel Resveratrol-Arsenic Trioxide Combination Treatment Synergistically Induces Apoptosis of Adriamycin-Selected Drug-Resistant Leukemia K562 Cells
}

Jing Chen ${ }^{*}$, Baoying Tian ${ }^{2}$, Cunmin Zhou ${ }^{1}$, Jingjing Sun ${ }^{1}$, Li Lin $^{1}$, Shucheng Jin ${ }^{1}$, Quanrui Liu ${ }^{1}$, Siyu Fu'

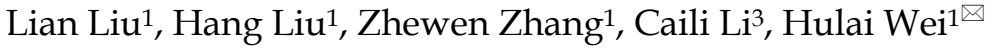

1. Key Laboratory of Preclinical Study for New Drugs of Gansu Province, School of Basic Medical Sciences, Lanzhou University, Lanzhou, Gansu, 730000

2. Hanzhong vocational and technical college, Hanzhong, Shanxi, 723000

3. School of Medicine of Northwest University for Nationalities, Lanzhou, Gansu 730030, P.R. China

* Contributed equally

$\square$ Corresponding author: Hulai Wei, MD, Lanzhou University, 199 Donggang West Road, Lanzhou, 730000, China. Tel: 0086-931-8915082; Email: weihulai@lzu.edu.cn

(1) The author(s). This is an open access article distributed under the terms of the Creative Commons Attribution License (https://creativecommons.org/licenses/by/4.0/). See http:/ /ivyspring.com/terms for full terms and conditions.

Received: 2019.02.28; Accepted: 2019.08.09; Published: 2019.08.29

\begin{abstract}
Leukemia cells can develop resistance to apoptosis induced by chemotherapeutic agents. Concomitant multidrug resistance of cells remains the greatest clinical obstacle in the effective treatment of blood and solid tumors. Natural products have been identified that possess the capacity to modulate chemotherapeutic resistance and induce apopotosis. In this study, we generated adriamycin-resistant K562 leukemia (K562/RA) cells and compared the responses of sensitive and resistant leukemia cells to the natural products arsenic trioxide (ATO) and resveratrol (Rsv), with a view to determining whether Rsv potentiates the sensitivity of drug-resistant cells to ATO-induced apoptosis and the associated molecular mechanisms. Our results showed that resistance of K562/RA cells induced by adriamycin treatment was significantly higher (115.81-fold) than that of parental K562 cells. Simultaneously, K562/RA cells were cross-resistant to multiple agents, with the exception of ATO. Rsv enhanced the sensitivity of K562/RA cells to ATO and reduced the required dose of ATO as well as associated adverse reactions by promoting the proliferation inhibitory and apoptosis-inducing effects of ATO, which may be associated with reduced expression of the drug resistance genes $\mathrm{mdrl} / \mathrm{P}$-gp, mrpl/MRPl and bcrp/BCRP, as well as the apoptotic inhibitory genes bcl-2, NF-KB and P53, and conversely, activation of caspase-3. Our collective findings indicate that ATO and Rsv synergistically enhance the sensitivity of drug-resistant leukemia cells to apoptosis.
\end{abstract}

Key words: leukemia, multidrug resistance, arsenic trioxide, resveratrol, apoptosis

\section{Introduction}

Long-term or repeated use of specific chemotherapy drugs can induce multidrug resistance of leukemia cells, even at therapeutic doses [1-3]. Reduced apoptosis-inducing activity of chemotherapy drugs is an important mechanism contributing to the development of multidrug resistance (MDR) in leukemia cells [4-6]. Identification of novel drugs that have no cross-resistance with conventional chemotherapy drugs and development of agents that enhance the apoptosis-inducing effect of currently available drugs are potentially effective measures to overcome MDR of leukemia. Arsenic 
trioxide (ATO) has good efficacy for many leukemia and solid tumor types with fewer side-effects than other agents [7-11]. In acute promyelocytic leukemia patients who achieve remission after retinoic acid treatment, combination therapy with ATO has been shown to consolidate the efficacy of retinoic acid. ATO is thus considered the 'gold partner' of retinoic acid [12,13]. Although results obtained with ATO provide an optimistic perspective in the management of leukemia, the compound exerts cytotoxicity, which causes damage to human organs. The majority of patients treated with ATO ( 75\%) experience grade 1 or 2 liver dysfunction [12-14]. In addition, a number of patients in remission after treatment with a combination of retinoic acid and ATO experience relapse. These patients often carry the missense mutations A216V, L218P, A216T, S214L, L217F and S220G in the promyelocytic leukemia protein-retinoic acid receptor a (PML/RARa) domain, which induce arsenic resistance, and consequently, a significant increase in mortality [12-14]. In vitro studies have confirmed a marked apoptosis-inducing effect of ATO on leukemia cells, especially those that are drug-resistant. However, divalent, trivalent and pentavalent arsenic intermediates have been detected in saliva, urine and blood of ATO-treated patients in clinical trials. The toxic effects of accumulated arsenic on the human liver, kidney and cardiovascular system cannot be overlooked [14-16].

Resveratrol (Rsv) is a natural antioxidant that not only has anti-viral and immune modulation properties but also prevents mutation and tumor development $[17,19]$. Rsv is reported to significantly reduce the malondialdehyde and $\mathrm{NO}$ contents in rat serum, liver, spleen, lung and brain induced by ATO, suggestive of effective antiperoxidase activity [20,21]. Rsv has been shown to enhance apoptosis of HeLa, MCF-7 and NB40 cells induced by ATO, both in vitro and in vivo. Moreover, Rsv protects normal cells against ATO toxicity by promoting apoptosis in tumor cells, although the precise mechanisms are currently unclear [22]. In this study, we generated drug resistance in K562 leukemia cells using adriamycin (ADM) and compared the responses of sensitive and resistant cell groups to ATO and Rsv. Additionally, the effects of combined treatment with ATO and Rsv on drug-resistant leukemia cells and the associated molecular mechanisms were investigated.

\section{Materials and Methods}

\section{Reagents}

SYBR Premix Ex Taq (RR820A) and Prime Script RT reagents (RR036A) were obtained from Takara Bio (Otsu, Japan). P-gp (mdr1), MRP1 (mpr1), BCRP (bcrp) and $\beta$-actin primers were additionally synthesized by Takara. Mouse anti- $\beta$-actin (3598.100, BioVision, Milpitas, CA, USA), rabbit anti-BCL-2 (12789-1-AP; Proteintec, Chicago, USA), rabbit anti-BAX (50599-2-lg; Proteintec), mouse anti-P53 (BM0101), rabbit anti-NF-kB (PB0161), rabbit anti-P-gp (BA1351-2), anti-BCRP (BA2307-2), anti-MRP1 (BA0567) (all from Boster, Wuhan, China), rabbit anti-cleaved caspase-3 (YM3431; ImmunoWay, Plano, TX, USA) and P-gp antibodies (MRK16, Mc-012; Kamiya Biomedical, Seattle WA, USA) were used. The chemotherapeutic drug ADM was purchased from Shenzhen Wanle Pharmaceutical Co. (Shenzhen, China) and ATO and Rsv acquired from Sigma-Aldrich (Merck KGaA, Darmstadt, Germany).

\section{Cell culture and incubation}

Human leukemia K562 cells were purchased from American Type Culture Collection (ATCC, Manassas, USA). Cells were maintained in RPMI 1640 medium (Gibco; Thermo Fisher Scientific, Inc. Waltham, MA, USA) supplemented with $10 \%$ fetal calf serum (Hyclone; GE Healthcare Life Sciences, Logan, UT, USA) and cultivated at $37^{\circ} \mathrm{C}$ in a $5 \% \mathrm{CO}_{2}$ incubator.

\section{Generation of ADM-resistant cells}

ADM resistance in human leukemia K562 cells was induced by long-term exposure to continuous stepwise increments of ADM. Cells were cryopreserved and recovered after 2 to 3 months of induction to prevent mutations until they were stably able to tolerate $16 \mu \mathrm{M}$ ADM. This procedure was used to generate a drug-resistant leukemia subline, designated K562/RA.

\section{In vitro drug sensitivity analysis}

A total of $0.8 \times 10^{5}$ target cells $/ \mathrm{ml}$ were plated in 96-well plates and cultured at $37^{\circ} \mathrm{C}$ with the specified concentrations of chemotherapeutic agents for 24-72 h. Absorbance was quantified using a Powerwave $X$ plate reader (Omega Bio-Tek, Inc., Norcross, GA, USA). The MTT assay (in which each well was incubated with $5 \mathrm{mg} / \mathrm{ml}$ MTT for $4 \mathrm{~h}$ at $37^{\circ} \mathrm{C}$, followed by overnight incubation with $100 \mu 110 \%$ SDS at $37^{\circ} \mathrm{C}$ ) was employed for determination of the half-maximal inhibitory concentration $\left(\mathrm{IC}_{50}\right)$ values for ADM and cytotoxicity assays.

\section{Morphological features of apoptotic cells}

Target cells were collected and stained with Giemsa-Wright stain (Solarbio, Beijing, China) according to the manufacturer's instructions. Morphologic changes were analyzed under an AX80 optical microscope (Olympus, Tokyo, Japan). 


\section{Flow cytometric analysis of caspase- 3 activity and apoptosis}

For the caspase-3 activity assay, cells were collected and labeled with FITC-DEVD-FMK (BioVision, Milpitas, CA, USA) for $30 \mathrm{~min}$, which irreversibly binds to activated caspase- 3 . Caspase- 3 activity was directly determined using an Epics XL-4 flow cytometer (Beckman-Coulter, Brea, CA, USA).

\section{Real-time quantitative RT-PCR}

Total cellular RNA was extracted using a TRIzol kit (Invitrogen; Thermo Fisher Scientific, Inc. Waltham, MA, USA) and cDNA was obtained by PrimeScript $^{\mathrm{TM}}$ RT reagent Kit with gDNA Eraser (RR047A, Takara Bio, Otsu, Japan). Amplification was performed with the following primers: MRP1, forward: 5'TGCAGAAGGCGGGGAGAACCTC3', reverse: 5'GTCGTCCGTTTCCAGGTCCACG3'; P-gp, forward:5'CCCATCATTGCAATAGCAGG3', reverse: 5'GTTCAAACTTCTGCTCCTGA3'; BCRP2, forward: 5'GCTGCAAGGAAAGATCCAAGT 3', reverse: 5'TAGTTGTTGCAAGCCGAAGAG 3', $\beta$-actin, forward: 5'-TGCTCCTCCTGAGCGCAAGTA-3', reverse: 5'-CCACATCTGCTGGAAGGTGGA-3'. The PCR conditions included initial denaturation at $95^{\circ} \mathrm{C}$ for $10 \mathrm{sec}, 40$ cycles of denaturation at $95^{\circ} \mathrm{C}$ for $5 \mathrm{sec}$ and annealing at $60^{\circ} \mathrm{C}$ for $30 \mathrm{sec}$. Reactions were conducted using a Rotor-Gene 3000 quantitative PCR amplifier (CobetteRes. Inc, Sydney, Australia). The relative mRNA levels were calculated by comparison to that of $\beta$-actin via the $2^{-\Delta \Delta \mathrm{Ct}}$ method [23].

\section{Western blot analysis}

Following lysis of target cells lysed using a radioimmunoprecipitation assay protein extraction reagent (P0013B; Beyotime Inc. Shanghai, China), proteins were fractionated via SDS-PAGE and transferred onto PVDF membranes (EMD Millipore, Billerica, MA, USA). Next, membranes were probed with primary antibodies and anti- $\beta$-actin antibody (Mouse $\mathrm{mAb}$; BioVision, Milpitas, CA, USA), followed by IRDye800CW-conjugated goat anti-mouse secondary antibodies (926-32210, dilution, 1:10,000, LI-COR Biosciences, Lincoln, NE, USA) or IRDye680DX-conjugated secondary antibodies (goat anti-rabbit, 926-32221, 1:10,000, LI-COR Biosciences). Antibody-coated protein bands in immunoblots were quantitated and visualized using an Odyssey double-color infrared-laser imaging system (Odyssey v1.2 software; LI-COR Biosciences).

\section{Statistical analysis}

All data are presented as means \pm standard deviation. Multiple comparisons among the three groups were performed using one-way analysis of variance (ANOVA). Intragroup comparisons were conducted using the paired Student's $t$ test. Error bars represent standard deviation, and $P<0.05$ indicates statistically significant differences between groups.

\section{Results}

\section{Generation of multidrug-resistant leukemia K562 cell strains}

Drug resistance and cross-resistance of $\mathrm{K} 562$

During the generation of ADM-induced resistance in K562 cells, tolerance of cells to ADM increased gradually with increasing concentrations and treatment durations. When cells could tolerate 16 $\mu \mathrm{M}$ ADM, $\mathrm{IC}_{50}$ values at 48 and $72 \mathrm{~h}$ for resistant cells were 84.2 and 94.2 times higher than that for parental cells, respectively (Figure 1A). Notably, these cells additionally developed resistance to other chemotherapeutic drugs including pirarubicin, daunorubicin, 5-FU, etoposide, vincristine and paclitaxel (Figure 1B), indicating that long-term and repeated stimulation with ADM induces acquisition of MDR in K562 cells. Interestingly, however, no significant cell resistance to ATO was evident (Figure 1C). K562 cells that tolerated $16 \mu \mathrm{M}$ ADM were designated K562/RA.

Expression of drug-resistant genes and proteins in K562 cells during ADM induction of resistance

Initially, we observed extremely low expression of mdr1/P-gp, mrp1/MRP and bcrp/ BCRP in K562 cells. During the development of ADM-induced resistance of K562 cells, expression of mdr1, mrp1 and bcrp genes increased with the tolerable ADM concentration (Figure 2A). In K562 cells able to tolerate $16 \mu \mathrm{M}$ ADM, expression of mdr1, mrp1 and brcp was 132.5-fold, 2.85-fold and 8.87-fold higher than that in parental cells, respectively. Flow cytometry (FCM) additionally showed an increase in the positive rate (PR) of P-gp protein expression and mean fluorescence indensity (MFI) with higher tolerable ADM concentrations. At a tolerable ADM concentration of $16 \mu \mathrm{M}$, nearly $100 \%$ cells displayed positive expression of P-gp (Figure 2B). Western blot data confirmed increased expression of P-gp, BCRP and MRP1 proteins in K562/RA cells. Notably, P-gp and MRP1 were almost undetectable in parental K562 but significantly upregulated in K562/RA cells (Figure 2C).

\section{Rsv enhanced the proliferation inhibitory activity of ATO on K562/RA cells}

After 24-72 h treatment with 0.5-8 $\mu \mathrm{M}$ ATO, proliferation rates of both K562/RA and parental K562 cells were significantly inhibited. The inhibitory 
rate was positively correlated with ATO concentration and treatment duration. Overall sensitivities of K562/RA and K562 cells to ATO were not significantly different (Figure 3A). After 24-72 h treatment with $10-80 \mu \mathrm{M}$ Rsv, proliferation activity of K562/RA and K562 cells was also inhibited, with no significant differences in tolerance of the two cell types to Rsv (Figure 3B). Upon a combination treatment of $2 \mu \mathrm{M}$ ATO and 20 or $40 \mu \mathrm{M}$ Rsv $(2 \mu \mathrm{M}$ ATO $+20 \mu \mathrm{M}$ Rsv and $2 \mu \mathrm{M}$ ATO $+40 \mu \mathrm{M}$ Rsv), the inhibitory rates in $\mathrm{K} 562 / \mathrm{RA}$ cells were $67.7 \%$ and $72.1 \%$ after $24 \mathrm{~h}, 76.7 \%$ and $88.6 \%$ after $48 \mathrm{~h}$ and $93.4 \%$ and $99.1 \%$ at $72 \mathrm{~h}$, respectively, compared to $28.8 \%$ and $39.4 \%$ after $24 \mathrm{~h}, 43 \%$ and $59.3 \%$ after $48 \mathrm{~h}$ and $65.2 \%$ and $77.7 \%$ at $72 \mathrm{~h}$, respectively, in K562 cells. These data demonstrate that the efficacy of combination therapy with ATO and Rsv is significantly higher in K562/RA than parental K562 cells (Figure 3C), which suggests that Rsv enhances the suppressive effect of ATO in resistant cells.

\section{Rsv enhances the apoptosis-inducing effect of ATO in K562/RA cells}

\section{Morphological changes of apoptosis}

We observed no significant morphological changes in K562 or K562/RA cells following treatment with $20 \mu \mathrm{M}$ Rsv. After 24-h treatment with 2 $\mu \mathrm{M}$ ATO or $2 \mu \mathrm{M}$ ATO plus $20 \mu \mathrm{M}$ Rsv, morphological changes characteristic of apoptosis, such as chromatin condensation and cell shrinkage, were observed, which were more pronounced in cells treated with the drug combination (Figure 4A).

\section{Caspase-3 activation}

FCM analysis showed that after 24-h treatment with $2 \mu \mathrm{M}$ ATO alone, the positive rate of caspase-3 activation in K562 and K562/RA groups increased from $2.13 \%$ and $2.26 \%$ in untreated control cells to $8.27 \%$ and $8.40 \%$, respectively. After treatment with $40 \mu \mathrm{M}$ Rsv alone, the positive rate increased to $22.19 \%$ and $16.78 \%$ while combined treatment with ATO and Rsv induced an increase in the rate of caspase- 3 activation to $48.7 \%$ and $81.93 \%$, respectively. Our results clearly indicate that both ATO and Rsv enhance caspase- 3 activity in resistant and sensitive K562 cell types. Moreover, treatment with the ATORsv combination promoted caspase-3 activity more significantly than either ATO or Rsv alone. This effect was more prominent in drug-resistant K562/RA cells (Figure 4B). The ATO and Rsv combination induced a significant increase in cleaved caspase-3 expression, particularly in K562/RA cells. These findings suggest that Rsv significantly enhances the

$\mathrm{C}$

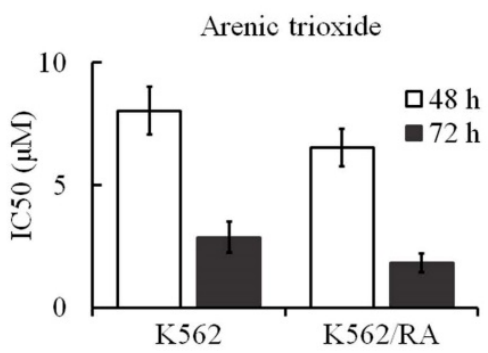

B

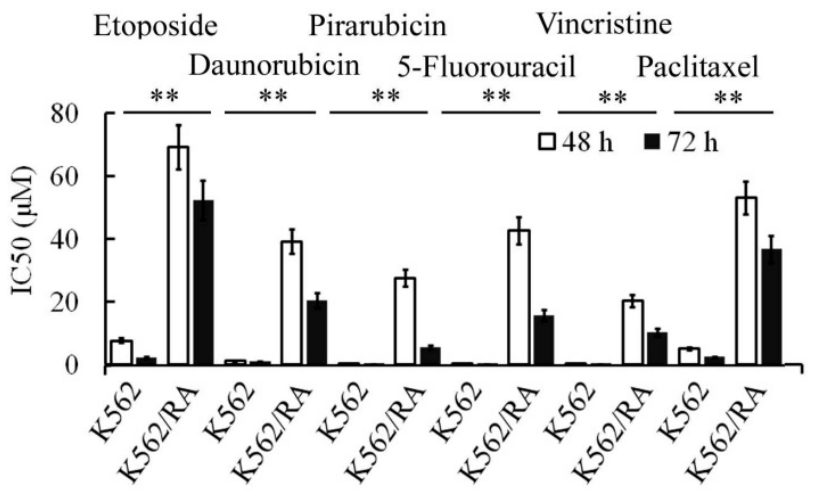

Figure 1. ADM promotes acquisition of multidrug resistance in $\mathrm{K} 562$ cells. After treatment, $\mathrm{K} 562$ cells developed resistance to $\mathrm{ADM}$ (A) in addition to other chemotherapeutic drugs, including pirarubicin, daunorubicin, vincristine, paclitaxel, etoposide and 5 -FU (B). However, sensitivity of cells to ATO was not significantly changed (C). $* P<0.05$, $* * P<0.01$, compared to $\mathrm{K} 562$ cells. 
apoptosis-inducing effect of ATO in drug-resistant cells (Figure 4C).

\section{BAX/BCL-2 expression}

Treatment with $2 \mu \mathrm{M}$ ATO or 20 or $40 \mu \mathrm{M}$ Rsv alone led to significant upregulation of BAX protein in K562 and K562/RA cells. Concomitantly, expression of BCL-2 was weakly downregulated. The BAX/BCL-2 ratios in K562 cells treated with $2 \mu \mathrm{M}$ ATO, $20 \mu \mathrm{M}$ Rsv and $40 \mu \mathrm{M}$ Rsv were 2.67-, 1.39- and 1.32-fold higher than that of the control group, respectively (Figure 5A-D). After combination treatment with $2 \mu \mathrm{M}$ ATO and $20 \mu \mathrm{M}$ or $40 \mu \mathrm{M}$ Rsv, the BAX/BCL-2 ratio was 2.42- and 7.7-fold higher than that of the control group for the K562 cell line. For the K562/RA cell line, the ratio was 20.27- and 97.33-fold higher in the treatment groups, respectively (Figure 5E). Our results suggest that Rsv enhances the apoptosis-inducing effect of ATO via concomitantly upregulating BAX and downregulating BCL-2. This synergistic apoptosis-promoting activity is more pronounced in drug-resistant K562/RA cells.

\section{P53 expression}

Treatment with $2 \mu \mathrm{M}$ ATO, $20 \mu \mathrm{M}$ or $40 \mu \mathrm{M}$ Rsv alone had no significant effects on P53 levels. However, expression of P53 was markedly decreased in K562 and K562/RA cells after combined treatment with ATO and Rsv (Figure 6A-D). In particular, co-treatment with $2 \mu \mathrm{M}$ ATO and $20 \mu \mathrm{M}$ or $40 \mu \mathrm{M}$ Rsv induced a more significant decrease in $\mathrm{P} 53$ expression in K562/RA cells (Figure 6A-D).
A
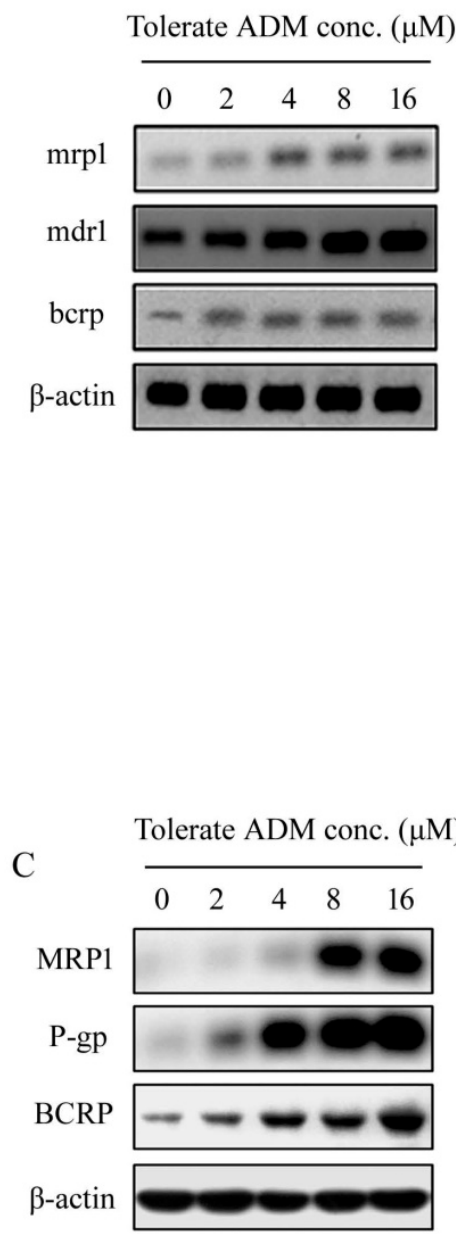

B

Tolerate ADM
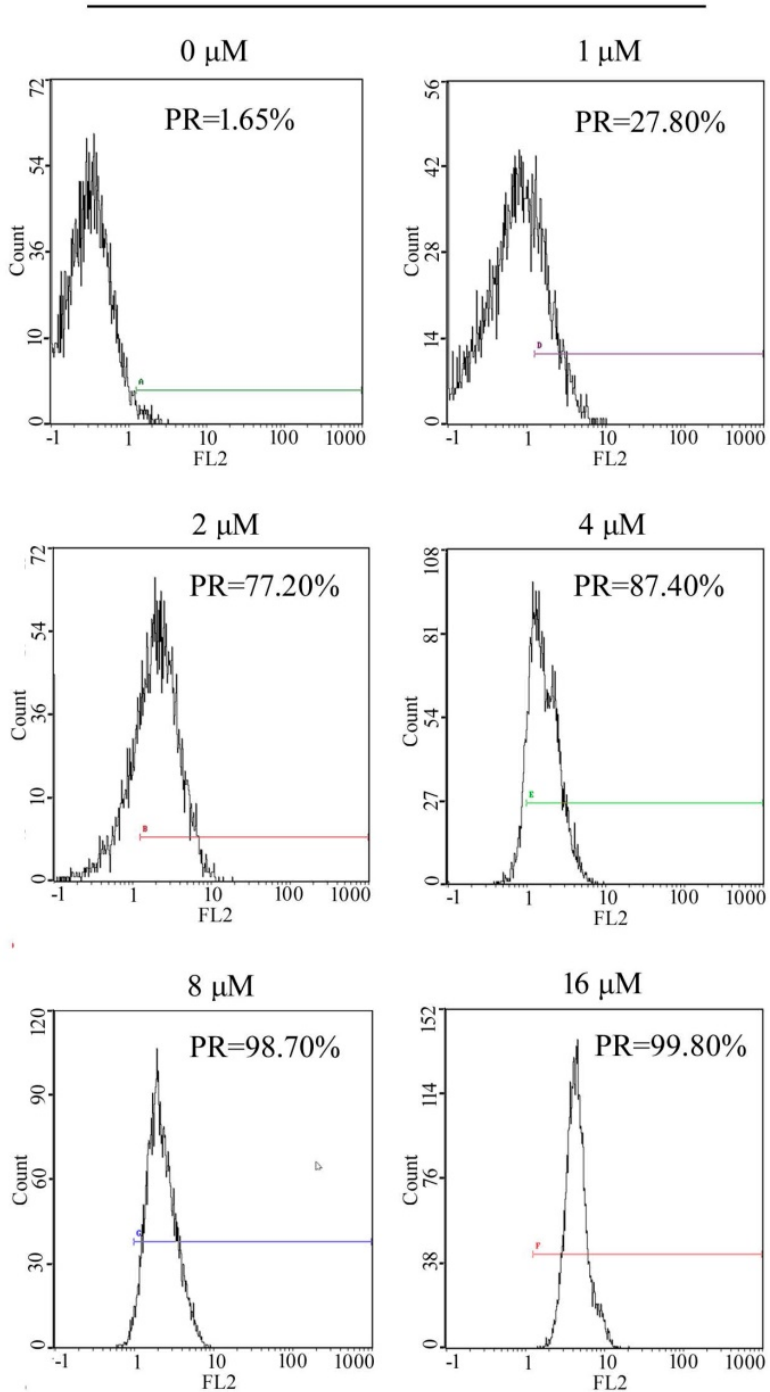

Figure 2. Expression of resistance-related genes and corresponding proteins in K562 cells tolerating different concentrations of ADM.Real-time RT-PCR analysis of relative expression of $m d r l$, mrpl and bcrp mRNA in K562 cells with ADM resistance (A). Positive expression rate (PR) of P-gP detected with flow cytometry (B). Western blot analysis of intracellular P-gP, MRPI and BCRP protein levels (C). 
A

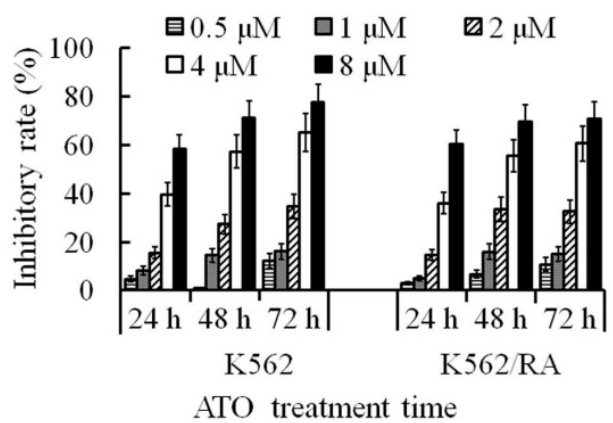

ATO treatment time
B

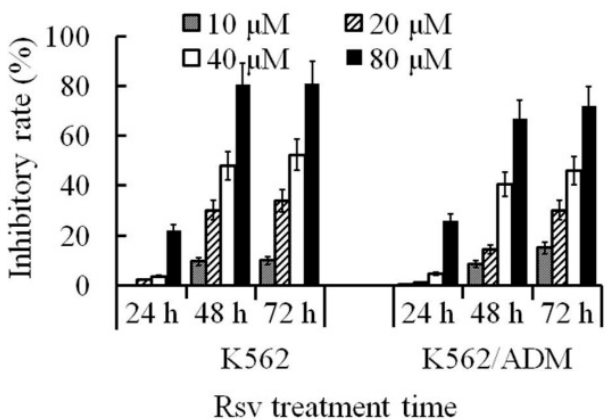

Rsv treatment time

C

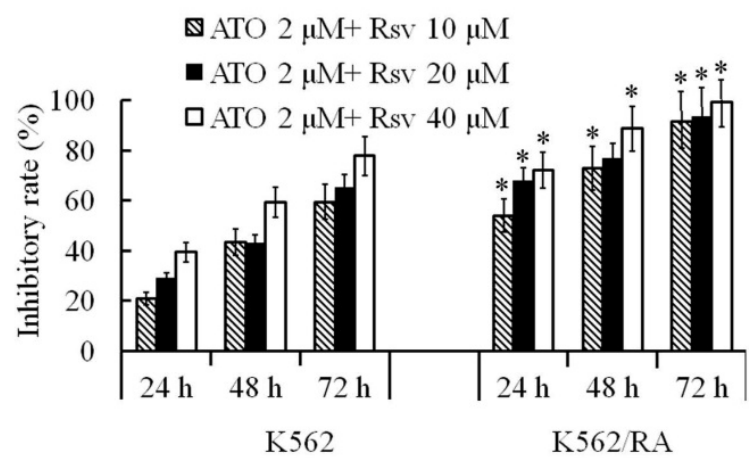

Figure 3. Inhibitory effects of ATO and Rsv on K562/RA cell proliferation. A, Comparison of the proliferation inhibitory rates of K562/RA and parental K562 cells after 24-72 $\mathrm{h}$ treatment with 0.5-8 $\mu \mathrm{M}$ ATO. B, Comparison of the proliferation inhibitory rates of K562/RA and parental K562 cells after 24-72 h treatment with 10-80 $\mu$ M Rsv. C, Inhibitory effects of a combination of $2 \mu \mathrm{M}$ ATO and $20 \mu \mathrm{M}$ or $40 \mu \mathrm{mol} / \mathrm{L}$ Rsv on proliferative capacity. $* P<0.05$, compared to K562 cells.

\section{NF-KB expression}

Activated NF-KB inhibits apoptosis. Treatment with $2 \mu \mathrm{M}$ ATO led to significant downregulation of NF-KB expression in K562/RA but exerted a weak effect on K562 cells (Figure 6A and B). Expression of NF-kB was not affected upon treatment with 20 or 40 $\mu \mathrm{M}$ Rsv. However, $2 \mu \mathrm{M}$ ATO combined with 20 or 40 $\mu \mathrm{M}$ Rsv induced downregulation of NF-KB in both K562 and K562/RA cell lines. In K562 cells, the combination treatment suppressed NF-KB expression to $63.03 \%$ and $20.61 \%$ relative to the control group, while in K562/RA cells, expression decreased to only $4.02 \%$ and $1.75 \%$ that of the control group (Figure $6 \mathrm{C}$ and D), suggesting that ATO and Rsv act synergistically to induce apoptosis in cells by inhibiting the NF- $\kappa \mathrm{B}$ signaling pathway, particularly in drug-resistant leukemia cells.

\section{Rsv enhances the inhibitory effect of ATO on expression of resistance-related genes in K562/RA cells}

Expression of mdr1, bcrp and mrp1 was high in ADM-induced resistant K562/RA cells but nearly undetectable in parental K562 cells (Figure 2). ATO (2 $\mu \mathrm{M})$ significantly inhibited expression of these genes in K562/RA cells $(21 \%, 33 \%$ and $36 \%$, compared to the control group, respectively). Rsv alone $(20 \mu \mathrm{M}$ or 40 $\mu \mathrm{M})$ had no or a weak inhibitory effect on the genes responsible for drug resistance, while combined treatment with $2 \mu \mathrm{M}$ ATO and 20 or $40 \mu \mathrm{M}$ Rsv synergistically downregulated the expression of drug-resistant genes in K562/RA cells. Levels of mdr1 were only $15 \%$ and $5 \%$ while those of bcrp were $30 \%$ and $25 \%$ relative to the control group, respectively, and $0.4 \%$ for mrp 1 , as detected with real time RT-PCR (Figure 7A and $\mathrm{B}$ ). Western blot results were in line with RT-PCR findings (Figure $7 C$ and D), further confirming that ATO combined with Rsv exerts a stronger apoptotic effect on K562/RA cells. The underlying mechanisms may be associated with downregulation of resistance-related genes through synergistic effects of ATO and Rsv.

\section{Discussion}

ATO has been widely used in the management of leukemia and shown to be effective in treating a variety of solid tumors, including lung, liver, ovarian, colon and esophageal cancer types [4,7-12]. The mechanisms underlying the anti-tumor activity of 
ATO are complex. ATO can induce tumor cell differentiation, promote degradation of PML/RARa fusion protein, block specific intracellular signaling pathways, and trigger apoptosis by activating the intracellular caspase cascade, downregulating Bcl-2 expression and inhibiting NF-kB. The apoptosis-inducing effect of ATO on leukemia and cancer cells represents the key mechanism that contributes to its anti-tumor activity [4,7-12,24]. In 1998 , Rsv was initially reported to induce apoptosis of human leukemia HL-60 cells via the Fas-FasL pathway with no apparent side-effects on peripheral lymphocytes $[25,26]$. Subsequent studies confirmed that Rsv exerts apoptotic effects on a variety of tumor cells, including T47D human breast cancer, U87 glioma, K562 leukemia, lymphoma and prostate cancer cells, with no significant side-effects on normal cells [22,25-29]. Notably, the compound has a strong killing effect on cancer stem cells. Additionally, previous experiments by our group have confirmed that Rsv induces apoptosis in leukemia K562 and stem cells.

A

$\begin{array}{lllll}\operatorname{ATO}(2 \mu \mathrm{M}) & - & + & - & + \\ \operatorname{Rsv}(20 \mu \mathrm{M}) & - & - & + & +\end{array}$

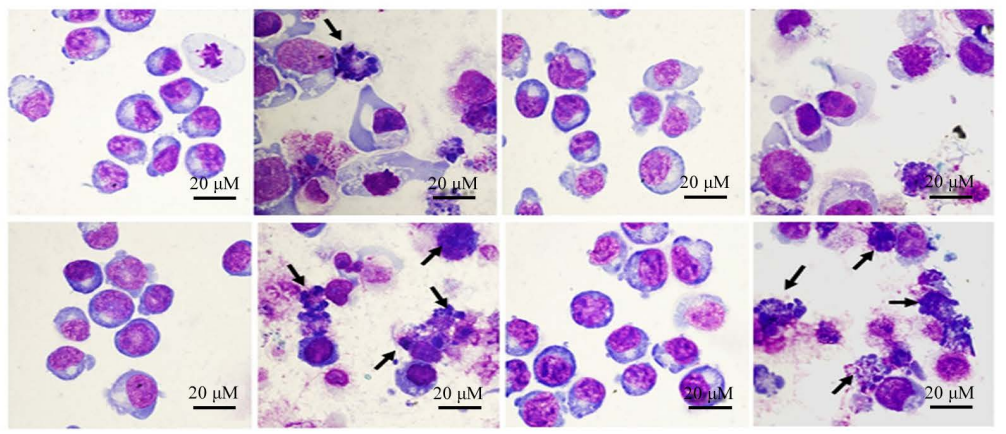

B

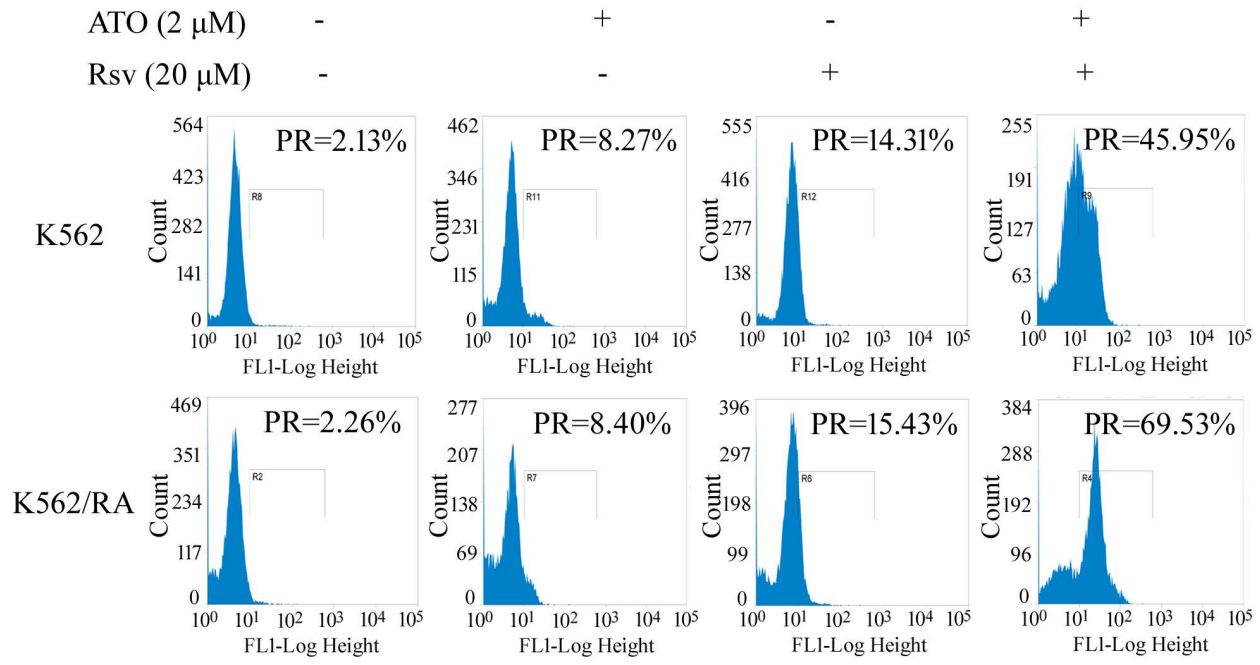

C

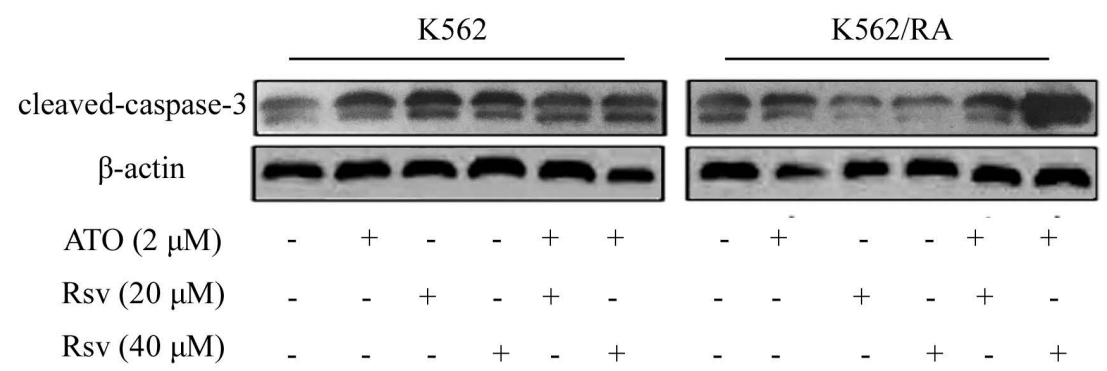

Figure 4. ATO combined with Rsv induces higher rates of K562/RA cell apoptosis. K562 or K562/RA cells were treated with ATO or Rsv alone or ATO combined with Rsv for $24 \mathrm{~h}$. A, Morphological changes observed under inverted light microscopy (100x) via Swiss-Giemsa staining. The arrowhead $(\rightarrow)$ indicates chromatin condensation, nuclear fragmentation and apoptotic bodies in apoptotic cells; B, FCM analysis of the positive rate (PR), mean fluorescence indensity (MFI) and of activated intracellular caspase-3; C, Western blot detection of cleaved caspase- 3 expression 
A

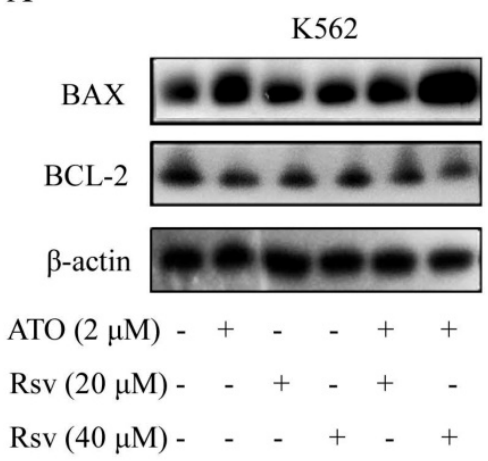

B

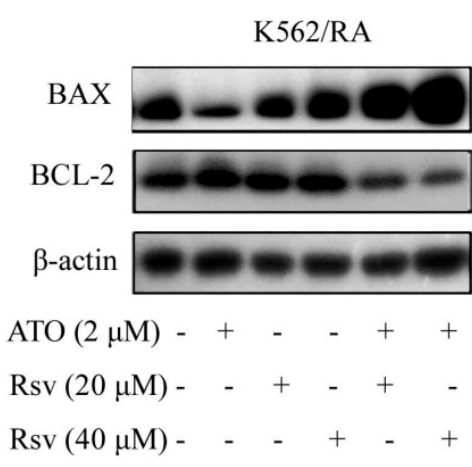

C

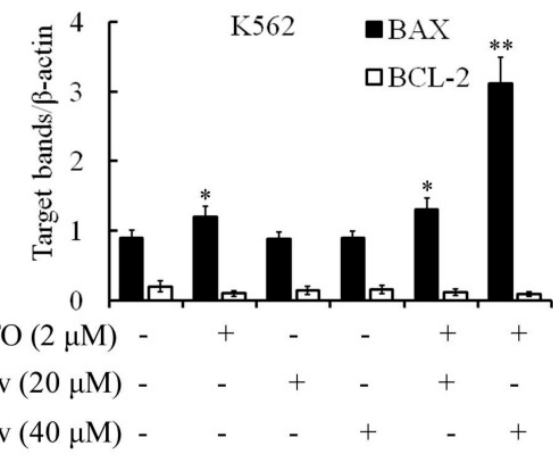

D

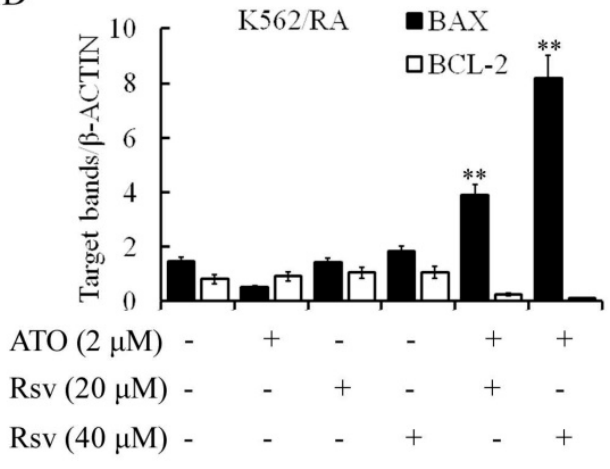

E

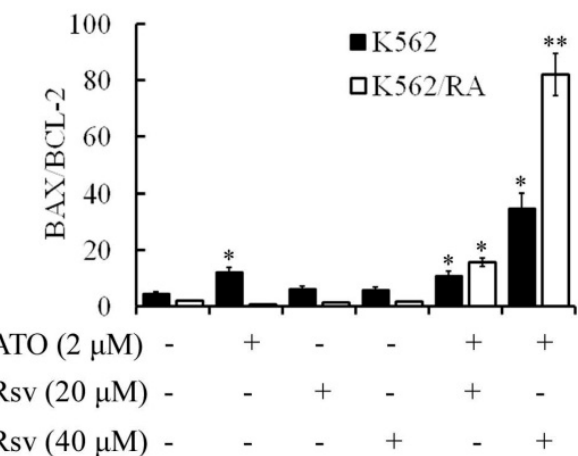

Figure 5. Effects of combination treatment with ATO and Rsv on BAX/BCL-2 expression.BAX and BCL-2 protein levels in K562 (A) and K562/RA (B) cells were detected via western blot after 24-h treatment with ATO or Rsv alone or a combination of ATO and Rsv. C, D, Corresponding relative protein levels of antibody-coated protein bands in immunoblots (scaned using an Odyssey double-color infrared-laser imaging system and their OD values were analyzed using an Odyssey v1.2 software. The relative protein levels

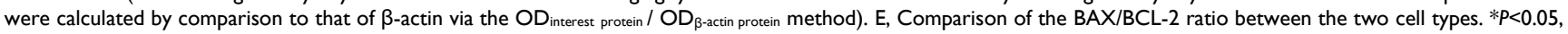
$* * P<0.01$, compared to control.

Recent studies suggest that Rsv not only has a synergistic inhibitory effect with ATO on tumor cells, but also reduces the toxicity of ATO alone. Here, we combined a lower concentration of ATO with Rsv to determine the proliferation inhibitory and apoptosis-inducing effects on resistant K562/RAcells. ATO, in combination with Rsv, exerted a synergistic inhibitory effect on proliferation of both K562/RA and parental K562 cells, which was more pronounced in the resistant cell line. The cells underwent morphological changes typical of apoptosis, and the apoptotic rate was significantly increased. Although caspase-3 expression was not affected to a marked extent, its activity was remarkably enhanced, particularly in K562/RA cells, indicating that Rsv enhances the proliferation inhibitory and apoptotic effects of ATO on K562/RA cells.

Interactions between BCL-2 on the mitochondrial membrane and BAX in the cytoplasm affect the stability of the mitochondrial membrane and determine cell apoptosis [28]. ATO combined with Rsv suppressed the expression of BCL-2 and P53 and increased the expression of BAX, thereby increasing the BAX/BCL-2 ratio. In drug-resistant K562/RA cells, Rsv and ATO exerted a stronger combined effect on downregulation of P53, which 
could trigger changes in the expression of downstream genes, such as BCL-2 and Bax. Accordingly, the permeability of the mitochondrial membrane was increased and $\mathrm{CytC}$ released, further activating caspase cascade reactions, leading to cellular apoptosis. NF- $\kappa \mathrm{B}$ is a transcription factor and the associated signaling pathway is inactive in normal cells but continuously activated in tumor cells. NF-KB activation leads to upregulation of the anti-apoptotic protein, BCL-2, which downregulates IкBa expression to further enhance activation of NF- $\kappa \mathrm{B}$ via a feedback mechanism. The transcription factor plays a critical role in the proliferation and apoptosis of tumor cells. We propose that ATO and Rsv synergistically inhibit the expression and activation of NF- $\mathrm{KB}$ in K562/RA cells, thus suppressing its promotory effect on BCL-2 expression to induce cell apoptosis.

The membrane transporters P-gp, MRP1 and BCRP protect cells against injury induced by chemotherapeutic drugs and other exotoxins. However, these proteins also mediate the development of multidrug resistance in tumor cells.
Meanwhile, evidence has shown that P-gp participates in the regulation of cellular apoptosis. In our experiments, the levels of mdr1, bcrp and mrp1 genes in K562/RA cells were significantly higher than those of parental K562 cells. Rsv did not affect expression of mdr1, bcrp and mrp1 in K562/RA cells but significantly enhanced the inhibitory effects of ATO on gene expression. These results suggest that Rsv and ATO synergistically enhance apoptosis of K562/RA cells, which may be associated with inhibition of mdr1, bcrp and mrp1. Additional studies [30] have shown that the promoter of mdr1 contains the binding site of NF- $\mathrm{kB}$. Binding of NF-kB activates the transcription of mdr1 and upregulates mdr1/P-gp. In tumor cells, mutated P53 can also activate the mdr1 promoter and upregulate the gene at the transcriptional level. Moreover, overexpression of BCL-2 promotes mdr1 expression. The collective findings support a complex regulatory network constituting apoptosis-related and drug-resistant genes that act in concert to modulate drug resistance and apoptosis of resistant leukemia cells.
A

K562

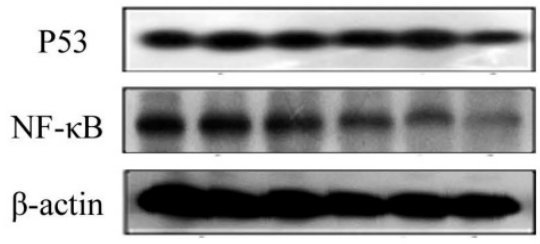

ATO $(2 \mu \mathrm{M})-\quad+\quad-\quad+\quad+$

$\operatorname{Rsv}(20 \mu \mathrm{M})-\quad-\quad+\quad+\quad-$

$\operatorname{Rsv}(40 \mu \mathrm{M})-\quad-\quad-\quad+\quad-\quad+$

B

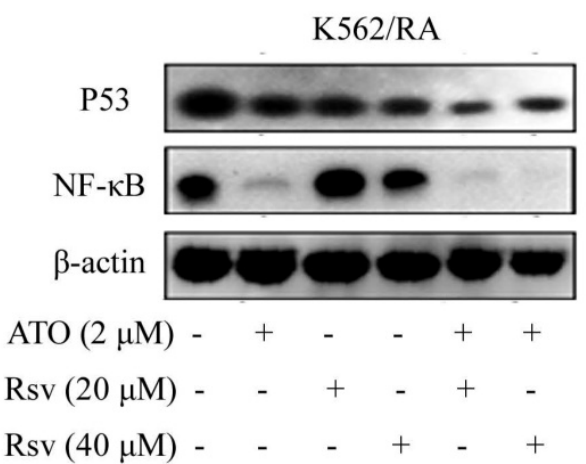

C

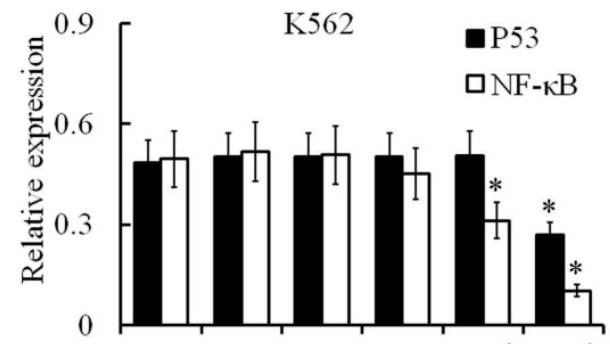

ATO $(2 \mu \mathrm{M})-\quad+\quad-\quad-\quad+\quad+$

$\operatorname{Rsv}(20 \mu \mathrm{M})-\quad-\quad+\quad-\quad+\quad-$

Rsv $(40 \mu \mathrm{M})$ - $\quad-\quad-\quad+\quad-\quad+$

$\mathrm{D}$

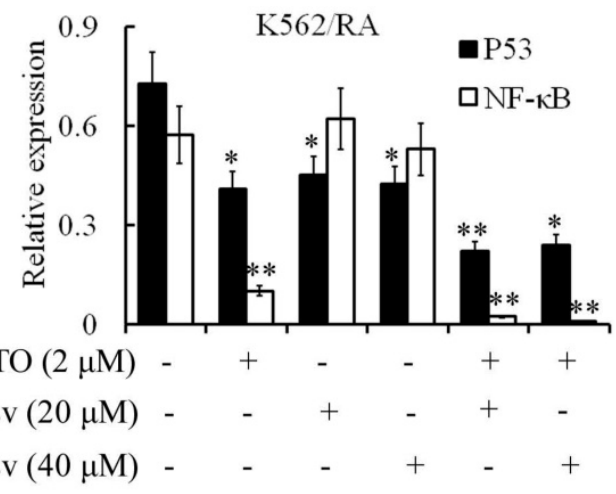

Figure 6. Combination treatment with ATO and Rsv inhibits P53 and NF-KB expression. After 24-h treatment with ATO or Rsv alone or ATO plus Rsv, western blot was applied to detect expression of P53 and NF-KB proteins in K562 (A) and K562/RA cells (B). C, D, Corresponding OD values. $* P<0.05$, $* * P<0.01$, compared to control. 
A

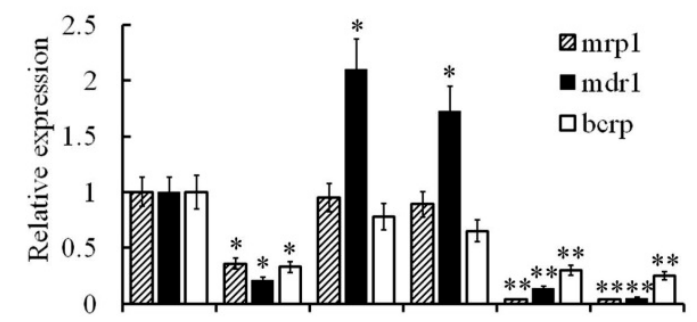

ATO $(2 \mu \mathrm{M})$ -

Rsv $(20 \mu \mathrm{M})$ -

Rsv $(40 \mu \mathrm{M})$ -
B

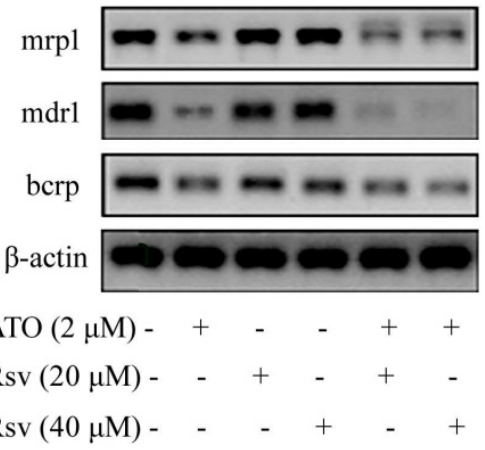

C

D
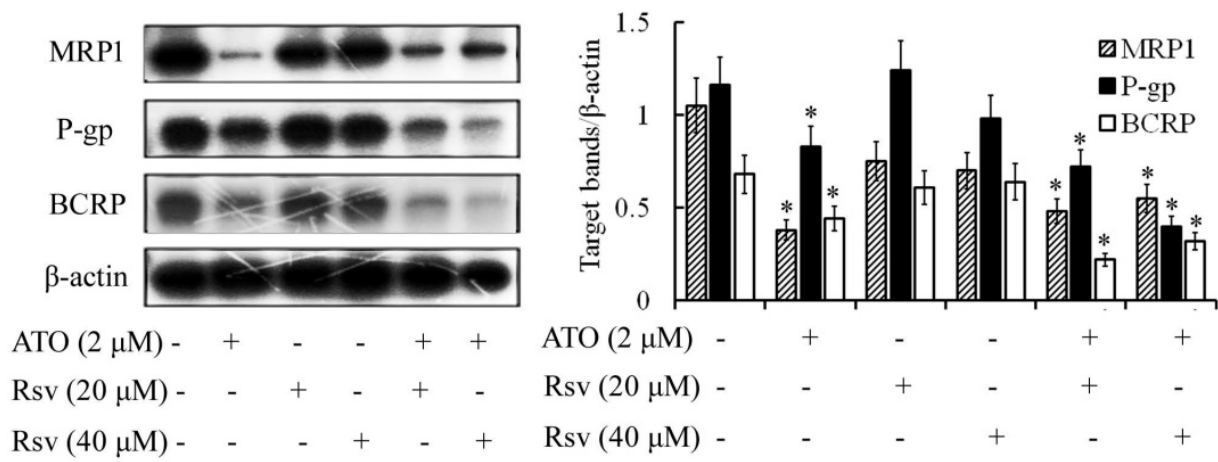

Figure 7. ATO and Rsv synergistically inhibit resistance-related genes. After 24-h treatment with ATO or Rsv alone or the combination of ATO and Rsv, real-time RT-PCR was used to detect expression of mdrl, mrpl and bcrp genes in K562 and K562/RA cells (A) and the corresponding electrophoresis spectrum of the amplified products (B). Western blot detection of expression of P-gp, MRPI and BCRP proteins (C) and the corresponding OD values (D). $* P<0.05$, $* * P<0.01$, compared to control.

\section{Conclusions}

Rsv enhances the apoptosis-inducing effect of ATO on both drug-resistant K562/RA and parental K562 cells and significantly suppresses expression of the resistance-related genes mdr1/P-gp, mrp1/MRP1 and bcrp/BCRP. Moreover, apoptosis induced by Rsv and ATO is associated with the Bcl-2/Bax and NF-kB pathways. Further research is warranted to elucidate the detailed mechanisms underlying the synergistic apoptotic effects of these drugs.

\section{Acknowledgements}

The authors thank International Science Editing (Shannon, Ireland) for language editing and revision of the manuscript.

\section{Funding}

This study was funded by the National Natural Science Foundation of China (grant nos. 81541025, 81141053 and 81660028), Fundamental Research Funds for the Central Universities (grant no. lzujbky-2016-174), Science and Technology Planning Project from Chengguan District, Lanzhou, Gansu
Province, China (grant no. 2016-7-11), and Natural Science Fund of Gansu (grant nos. 1208RJZA183 and 1208RJZA190).

\section{Author Contributions}

W.H.L. conceived and designed the study, revised the paper critically for important content and approved the final version for submission; C.J. and T.B. designed the study, performed the experiments, acquired and analyzed data and wrote the article; Z.C.M., S.J.J., L.L., J.S.C., L.Q.R., Z.M., L.L., L.H., and Z.Z.W. took part in performing experiments, interpretation of data, drafting and revision of the manuscript; X.B. and L.C.L. contributed reagents/materials and tools.

\section{Competing Interests}

The authors have declared that no competing interest exists.

\section{References}

1. Zhang QH, Dou HT, Xu P,Liu PS. Tumor recurrence and drug resistance properties of side population cells in high grade ovary cancer. Drug Res. 2014; 65:153-157. 
2. Gao F, Dong W, Yang W, Liu J, Zheng Z, Sun K. Expression of P-gp in acute myeloid leukemia and the reversal function of $\mathrm{As}_{2} \mathrm{O}_{3}$ on drug resistance. Oncol Lett. 2015; 9:177-182.

3. Matsumoto T, Jimi S, Hara S, Takamatsu Y, Suzumiya J, Tamura K. Importance of inducible multidrug resistance 1 expression in HL-60 cells resistant to gemtuzumab ozogamicin. Leuk Lymphoma. 2012; 53:1399-1405.

4. Kanjer K, Tatić S, Netić Sć-Konstantinović Z, Rabi ZA, Nikolić-Vukosavljević D. Treatment response to preoperative anthracycline-based chemotherapy in locally advanced breast cancer: the relevance of proliferation and apoptosis rates. Pathol Oncol Res. 2013; 19: 577-588.

5. Pommier Y, Sordet O, Antony S, Hayward RL, Kohn KW. Apoptosis defects and chemotherapy resistance: molecular interaction maps and networks. Oncogene. 2004; 23: 2934-2949.

6. Gutiérrez-González A, Belda-Iniesta C, Bargiela-Iparraguirre J, Dominguez G, García AP, Perona R, Sanchez-Perez I. Targeting Chk2 improves gastric cancer chemotherapy by impairing DNA damage repair. Apoptosis. 2013; 18: 347-360.

7. Sun RC, Board PG, Blackburn AC. Targeting metabolism with arsenic trioxide and dichloroacetate in breast cancer cells. Mol Cancer. 2011; 10: 142-156.

8. Yu J, Qian H.L, Li YF, Wang Y, Zhang XY, Liang X, Fu M, Lin C. Arsenic trioxide (As2O3) reduces the invasive and metastatic properties of cervical cancer cells in vitro and in vivo. Gynecol Oncol. 2007; 106: 400-406.

9. Pettersson HM, Pietras A, Persson MM, Karlsson J, Johansson L, Shoshan MC. Arsenic trioxide is highly cytotoxic to small cell lung carcinoma cells. Mol Cancer Ther.2009; 8:160-170.

10. Zhao H, Guo W, Peng C, Ji T, Lu XC. Arsenic trioxide inhibits the growth of adriamycin resistant osteosarcoma cells through inducing apoptosis. Mol. Biol. Rep. 2010; 37: 2509-2515.

11. Meister MT, Boedicker C, Graab U, Hugle M, Hahn H, Klingebiel T, Påhlman $\mathrm{S}$. Arsenic trioxide induces noxa-dependent apoptosis in rhabdomyosarcoma cells and synergizes with antimicrotubule drugs. Cancer Lett. 2016; 381: 287-295.

12. Tomita A, Kiyoi H, Naoe T. Mechanisms of action and resistance to all-trans retinoic acid (ATRA) and arsenic trioxide $\left(\mathrm{As}_{2} \mathrm{O}_{3}\right)$ in acute promyelocytic leukemia. Int J Hematol. 2013; 97:717-725.

13. Lo-Coco F, Avvisati G, Vignetti M, Thiede C, Orlando SM, Iacobelli S, Ferrara F. Retinoic acid and arsenic trioxide for acute promyelocytic leukemia. N Engl J Med. 2013; 369:111-121.

14. Zhu HH, Qin YZ, Huang XJ. Resistance to arsenic therapy in acute promyelocytic leukemia. N Engl J Med. 2014; 370:1864-1866.

15. Marasca R, Zucchini P, Galimberti S, Leonardi G, Vaccari P, Donelli A, Luppi M, Petrini M, Torelli G. Missense mutations in the PML/RARalpha ligand binding domain in ATRA-resistant $\mathrm{As}_{2} \mathrm{O}_{3}$ sensitive relapsed acute promyelocytic leukemia. Haematologica. 1999; 84: 963-968.

16. Mandal BK, Suzuki KT. Arsenic round the world: a review. Talanta. 2002; 58: 201-235.

17. Aschemann-Witzel J, Grunert K.G. Resveratrol and health from a consumer perspective: perception, attitude, and adoption of a new functional ingredient. Ann N Y AcadSci. 2015; 1348: 171-179.

18. Kim KO, Park H, Kim H.S. Effects of high-protein diet and/or resveratrol supplementation on the immune response of irradiated rats. Prev Nutr Food Sci. 2014; 19: 156-163.

19. Frazzi R, Tigano M. The multiple mechanisms of cell death triggered by resveratrol in lymphoma and leukemia. Int J Mol Sci. 2014; 15: 4977-4993.

20. Cristina G, Sara C, Roman K, Cruz G, Lisa R, Sergio DP, Elena Varaa, Jesús AFT. Protective effect of resveratrol against inflammation, oxidative stress and apoptosis in pancreas of aged SAMP8 mice. Exp Gerontol. 2017; 90: 61-67.

21. Yan F, Sun $\mathrm{X}, \mathrm{Xu}$ C. Protective effects of resveratrol improve cardiovascular function in rats with diabetes. Exp Ther Med. 2018; 15: 1728-1734.

22. Zhao XY, Yang S, Chen YR, Li PC, Dou MM, Zhang J. Resveratrol and arsenic trioxide act synergistically to kill tumor cells in vitro and in vivo. PLoS One. 2014; 9:1-9.

23. Livak KJ, Schmittgen TD. Analysis of relative gene expression data using real-time quantitative PCR and the 2(-Delta Delta C(T)) Method. Methods. 2001; 25:402-408

24. Zhao D, Jiang Y, Dong X, Liu Z, Qu B, Zhang Y, Ma N, Han Q. Arsenic trioxide reduces drug resistance to adriamycin in leukemic K562/A02 cells via multiple mechanisms. Biomed. Pharmacother. 2011; 65: 354-360.

25. Clement MV, Hirpara JL, Chawdhury SH, Pervaiz S. Chemopreventive agent resveratrol, a natural product derived from grapes, triggers CD95signaling-dependent apoptosis in human tumor cells. Blood. 1998; 92: 996-1001.

26. Leonard SS, Xia C, Jiang BH, Stinefelt B, Klandorf H, Harris GK, Shi X. Resveratrol scavenges reactive oxygen species and effects radical induced cellular responses. Biochem. Biophys. Res Commun. 2003; 309:1017-1026.

27. Chen J, Wei HL, Xie B, Wang B, Cheng J, Cheng J. Endoplasmic reticulum stress contributes to arsenic trioxide-induced apoptosis in drug-sensitive and -resistant leukemia cells. Leukemia Res. 2012; 36:1526-1535.

28. Edlich F. BCL-2 proteins and apoptosis: recent insights and unknowns. Biochem Biophys Res Commun. 2018; 500: 26-34.

29. Jara P, Spies J, Cárcamo C, Arancibia Y, Vargas G, Martin C, Salas M, Otth C, Zambrano A. The effect of resveratrol on cell viability in the burkitt's lymphoma cell line ramos. Molecules. 2017; 23:1-12.

30. Bourguignon LYW, Xia WL, Wong G. Hyaluronan-mediated CD44 Interaction with p300 and SIRT1 Regulates $\beta$-Catenin Signaling and NFkB-specific
Transcription Activity Leading to MDR1 and Bcl-xL Gene Expression and Chemoresistance in Breast Tumor Cells. J Biol Chem. 2008; 284: 2657-2671. 